EPJ Web of Conferences 59, 02015 (2013)

DOI: $10.1051 /$ epjconf/20135902015

(C) Owned by the authors, published by EDP Sciences, 2013

\title{
Illumination uniformity of capsules directly driven by a facility with thousands of laser beams
}

\author{
M. Temporal ${ }^{1, a}$, B. Canaud ${ }^{2}$ and V. Brandon ${ }^{2}$ \\ ${ }^{1}$ Universidad Politecnica de Madrid, 28040 Madrid, Spain \\ ${ }^{2}$ CEA, DAM, DIF, 91297 Arpajon, France
}

\begin{abstract}
The uniformity of the illumination of a spherical capsule directly driven by laser beams has been assessed numerically. Different schemes characterized by $\mathrm{N}_{\mathrm{D}}=12,20,32,48$ and 60 directions of irradiation associated to a single laser beam or a bundle of $\mathrm{N}_{\mathrm{B}}$ laser beams have been considered. The laser beams intensity profile is assumed super-Gaussian and the calculations take into account for beam imperfections as power imbalance and pointing errors. These facilities are characterized by a large number of laser beams $\mathrm{N}_{\mathrm{tot}}=\mathrm{N}_{\mathrm{D}} x \mathrm{~N}_{\mathrm{B}}$. Assuming relatively small laser beams with an energy of a few hundred of $\mathrm{J}$ (200 J-500 J) it turn out that a few thousand of beamlets (1000-5000) are needed in order to provide a total energy of 0.5-1 MJ. A large parametric study has been performed showing that the optimum laser intensity profile, which minimizes the root-mean-square deviation of the capsule illumination, depends on the values of the power imbalance and pointing error. Moreover, when beams imperfections are taken into account it is found that the uniformity of the illumination is almost the same for all facilities and only depends on the total number of laser beams $\mathrm{N}_{\text {tot }}$.
\end{abstract}

\section{INTRODUCTION}

In Direct Drive (DD) [1, 2] Inertial Confinement Fusion (ICF) [3-5] a spherical capsule is directly irradiated by laser beams. As a consequence of the fast laser energy deposition the capsule implodes and compresses the thermonuclear Deuterium-Tritium (DT) fuel. In order to provide a successful implosion, irradiation of the capsule must be highly uniform preventing the dangerous growth of RichtmyerMeshkov [6] and Rayleigh-Taylor [7] hydrodynamic instabilities.

Several partially overlapping laser beams characterized by irradiation directions [8-10] distributed to maximize the isotropy are usually considered to provide a highly uniform capsule irradiation. The pulse power driving a direct drive capsule implosion is assembled by a low-power $(\approx T W)$ pre-pulse followed by a relatively high power (tens of TW) main pulse. The laser pre-pulse generates the imprint phase. In this phase the expansion of the plasma corona does not affect the laser reflection and the uniformity of the energy deposition could be analyzed assuming a direct illumination of the capsule [11]. The laser beams assembled in a facility are affected by unavoidable imperfections as power imbalance and pointing error [11-18]. In this paper we analyze the illumination uniformity provided by five irradiation configurations characterized by $\mathrm{N}_{\mathrm{D}}=12,20,32,48$ and 60 irradiation directions [12]. It is shown that the optimal focal spot shape depends on the beam imperfections [13] and that taking beam imperfection into account the illumination uniformity is degraded by about one order of magnitude [11]. Finally, it is shown that the use of bundles [14, 15] of $\mathrm{N}_{\mathrm{B}}$ beamlets (laser beam unit) associated to each irradiation direction introduces a stochastic homogenization of the laser intensity which benefits the irradiation uniformity $[16,17]$.

\footnotetext{
ae-mail: mauro.temporal@hotmail.com
}

This is an Open Access article distributed under the terms of the Creative Commons Attribution License 2.0, which permits unrestricted use, distribution, and reproduction in any medium, provided the original work is properly cited. 
Table 1. Minimum intrinsic non-uniformity $\sigma_{0}$ and non-uniformities $\sigma_{1}$ for the five configurations.

\begin{tabular}{|l|c|c|c|c|}
\hline $\mathrm{N}_{\mathrm{D}}$ & $\Delta$ & $\mathrm{m}$ & $\sigma_{0}[\%]$ & $\sigma_{1}[\%](\delta)$ \\
\hline 12 & 0.80 & 2.8 & 0.39 & $4.15(0.96)$ \\
\hline 20 & 0.80 & 2.8 & 0.18 & $3.20(0.74)$ \\
\hline 32 & 0.80 & 3.0 & 0.15 & $2.72(0.61)$ \\
\hline 48 & 0.83 & 4.2 & 0.16 & $2.20(0.50)$ \\
\hline 60 & 0.80 & 3.0 & 0.08 & $1.95(0.43)$ \\
\hline
\end{tabular}

\section{ILLUMINATION UNIFORMITY}

The illumination uniformity has been calculated as the root-mean-square (rms) deviation $\sigma=\left[\int(I-\right.$ $\left.\underline{\mathrm{I}})^{2} \mathrm{~d} \Omega\right]^{1 / 2} /\left[(4 \pi)^{1 / 2} \mathrm{I}\right]$ of the laser intensity, where $\underline{\mathrm{I}}$ is the average intensity and the integral covers the whole surface of the unitary sphere. The laser intensity profile is assumed super-Gaussian $\mathrm{I}(\mathrm{r})=\mathrm{I}_{0} \exp \left[-(r / \Delta)^{m}\right]$, characterized by the parameters $\Delta$ (half-width at half maximum) and the exponent $\mathrm{m}$.

For any irradiation configuration it is possible to calculate the intrinsic non-uniformity $\sigma_{0}$ as a function of the laser focal spot parameters $\Delta$ and $\mathrm{m}$. The intrinsic non-uniformity assumes perfectly balanced and centered laser beams and characterizes an ideal configuration. A parametric study shows that a given irradiation configuration exhibits one or more minima of $\sigma_{0}$ in the space $[\Delta, \mathrm{m}]$. In Table 1 are shown the parameters $[\Delta, \mathrm{m}]$ that provide a minimum of $\sigma_{0}$ for five irradiation configurations. In the Table 1 are also shown the illumination uniformities $\sigma_{1}[\Delta, \mathrm{m}]$ evaluated assuming a power imbalance $\sigma_{\mathrm{PI}}=5 \%$ and a pointing error $\sigma_{\mathrm{PE}}=5 \%$. These non-uniformities $\sigma_{1}$ represent the average value of the non-uniformity evaluated on one thousand of calculations where random power imbalance and the pointing errors follow Gaussian distributions. To these average values $\sigma_{1}$ are associated a standard deviation $\delta$ which is also shown in Table 1 . As shown in Table 1 the inclusion of the beam imperfections increases the non-uniformity from $\sigma_{0}$ to $\sigma_{1}$ by about one order of magnitude.

In order to reduce the growth of the non-uniformity $\sigma_{1}$ as a consequence of the beam imperfections, it was proposed to associate at each irradiation direction a bundle of $\mathrm{N}_{B}$ independents beamlets. In this way the action of the $\mathrm{N}_{\mathrm{B}}$ beamlets provide a stochastic homogenization of the irradiation uniformity. Figure 1 shows the illumination uniformity evaluated for a facility characterized by $\mathrm{N}_{\mathrm{D}}=32$ irradiation directions. Frame (a) shows the intrinsic illumination non-uniformity $\sigma_{0}$ (neglecting any beam imperfections) as a function of the laser intensity profile parameters $\Delta$ and $\mathrm{m}$. In the other frames are shown the results accounting for the beam imperfections (power imbalance $\sigma_{\mathrm{PI}}=5 \%$ and pointing error $\sigma_{\mathrm{PE}}=5 \%$ ) and assuming one beamlet per irradiation direction (b), 9 beamlets (c) and 96 beamlets (d). As shown in figure 1 the optimum of the laser intensity profile (frame a) moves at larger $\Delta$ and higher $\mathrm{m}$ when the beam imperfections are taken into account (frames b). Moreover, these non-uniformities tend to reproduce the optimum intrinsic non-uniformity $\sigma_{0}$ when the number of beams is increased (from $\mathrm{N}_{\mathrm{B}}=1,9$ until 96). This is because errors associated to the whole bundle are reduced $\left(\approx 1 / \mathrm{N}_{\mathrm{B}}^{1 / 2}\right)$ as the number of beamlets increases, and tends to zero as $\mathrm{N}_{\mathrm{B}}$ tends to infinity.

In figure 2 are shown the optimum of the intrinsic non-uniformity $\sigma_{0}$ (stars) for five configurations characterized by $\mathrm{N}_{\mathrm{D}}=12,20,32,48$ and 60 . In the same figure are also shown the average nonuniformities calculated taking into account the beam imperfections (power imbalance $\sigma_{\mathrm{PI}}=5 \%$, pointing error $\sigma_{\mathrm{PE}}=5 \%$ ) and assuming a total number of beamlets $\mathrm{N}_{\text {tot }}=\mathrm{N}_{\mathrm{D}} \mathrm{XN}$. Three cases have been considered: $\mathrm{N}_{\text {tot }}=\mathrm{N}_{\mathrm{D}}$ (solid circles), $\mathrm{N}_{\text {tot }} \approx 300$ (empty circles) and $\mathrm{N}_{\text {tot }} \approx 1000$ (squares). The shadowed areas indicate the dispersion of one standard deviation $( \pm \delta)$. As it is shown in figure 2 considering one beamlet per irradiation direction $\left(\mathrm{N}_{\mathrm{B}}=1\right.$, solid circles) the non-uniformity decreases as the number of irradiation directions $\left(\mathrm{N}_{\mathrm{D}}\right)$ increases, nevertheless the non-uniformities are always higher than $1 \%$ for all five facilities. Differently, increasing the number of beamlets $\left(\mathrm{N}_{\mathrm{B}}\right)$ and assuming 

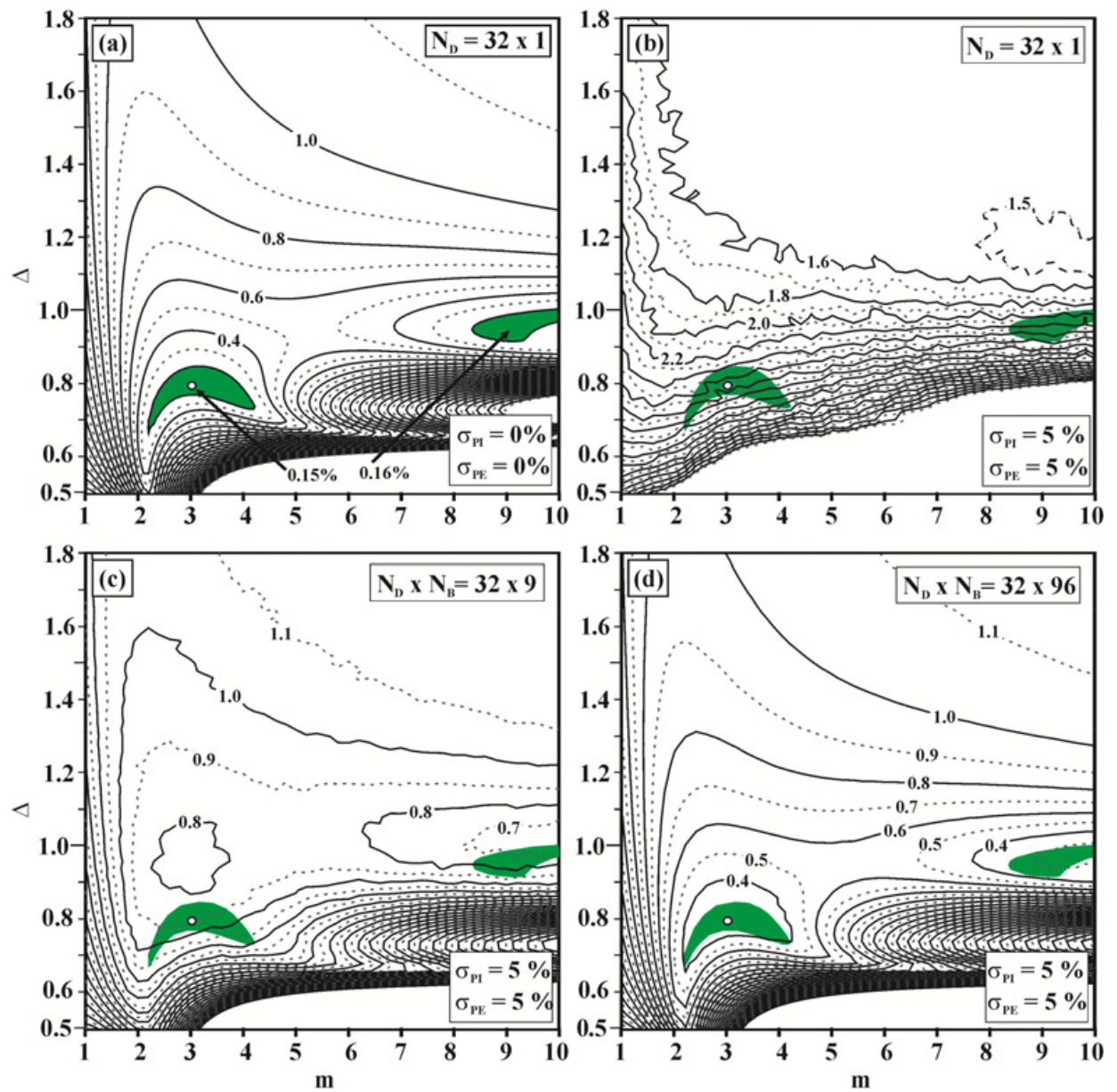

Figure 1. Illumination non-uniformities as a function of the laser intensity profile $[\Delta, \mathrm{m}]$.

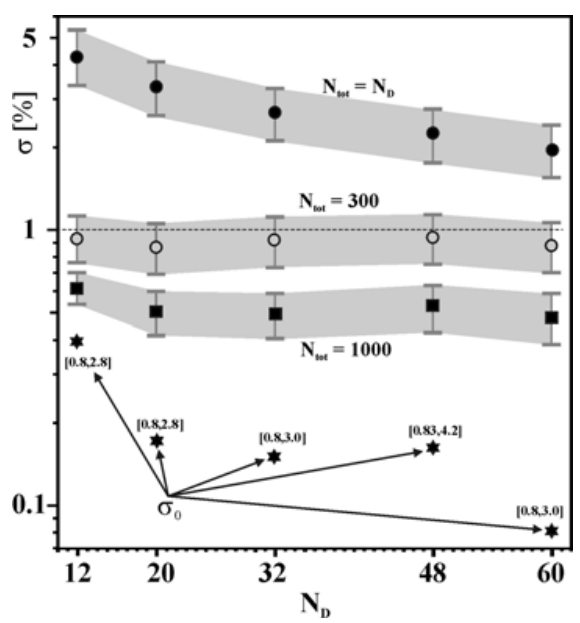

Figure 2. Illuminations uniformity as a function of the number of irradiation directions $\mathrm{N}_{\mathrm{D}}=12,20,32,48$ and 60. The results refer to a total number of beamlets $\mathrm{N}_{\text {tot }}=\mathrm{N}_{\mathrm{D}} \mathrm{x} \mathrm{N}_{\mathrm{B}}=\mathrm{N}_{\mathrm{D}}, \mathrm{N}_{\mathrm{tot}} \approx 300$ and $\mathrm{N}_{\text {tot }} \approx 1000$. 
the same total number of beamlets $\mathrm{N}_{\text {tot }} \approx 300$ or $\mathrm{N}_{\text {tot }} \approx 1000$ the non-uniformity is almost constant and independent of the number of directions of irradiation $\mathrm{N}_{\mathrm{D}}$.

\section{CONCLUSIONS}

The illumination uniformity has been calculated for five irradiation configurations. Assuming a power imbalance of $5 \%$ and a pointing error of $5 \%$ the non-uniformities $\sigma_{1}$ increase by about one order of magnitude by comparison with the intrinsic non-uniformities $\sigma_{0}$. To overcome this negative effect it has been proposed to associate to each direction of irradiation a bundle of $\mathrm{N}_{\mathrm{B}}$ independents beamlets. This is a natural way in order to reduce the imperfections associated to the whole bundle. It is found that the illumination uniformity scales as $1 / \mathrm{N}_{\text {tot }}^{1 / 2}$ and is given $[16,17]$ by $\sigma\left(\mathrm{N}_{\text {tot }}\right)=\left[\sigma_{0}^{2}+\mathrm{N}_{\mathrm{D}}\left(\sigma_{1}^{2}-\sigma_{0}^{2}\right) / \mathrm{N}_{\text {tot }}\right]^{1 / 2}$, where $\mathrm{N}_{\mathrm{D}}$ is the number of irradiation directions.

\section{References}

[1] B. Canaud, X. Fortin, F. Garaude, C. Meyer, F. Philippe, M. Temporal, S. Atzeni, and A. Schiavi, Nucl. Fusion 44, 1118 (2004)

[2] B. Canaud, F. Garaude, C. Clique, N. Lecler, A. Masson, R. Quach, and J. Van der Vliet, Nucl. Fusion 47, 1652 (2007)

[3] J. H. Nuckolls, L. Wood, A. Thiessen, and G. B. Zimmermann, Nature 239, 129 (1972)

[4] J. D. Lindl, P. Amendt, R. L. Berger, S. G. Glendinning, S. H. Glenzer, S. W. Haan, R. L. Kauffman, O. L. Landen, and L. J. Suter, Phys. Plasmas 11, 339 (2004); J. Lindl, Phys. Plasmas 2, 3933 (1995)

[5] S. Atzeni and J. Meyer-ter-Vehn, The Physics of Inertial Fusion (Oxford University Press, Oxford, 2004)

[6] R. D. Richtmyer, Commun. Pure Appl. Math. 13, 297 (1960); E. E. Meshkov, Fluid Dyn. 4, 101 (1969)

[7] Lord Rayleigh, Scientific Papers (Dover, New York, 1965); G. I. Taylor, Proc. R. Soc. London, Ser. A 201, 192 (1950)

[8] S. E. Bodner, J. Fusion Energy 1, 221 (1981)

[9] S. Skupsky and K. Lee, J. Appl. Phys. 54, 3662 (1983)

[10] A. J. Schmitt, Appl. Phys. Lett. 44, 399 (1984)

[11] M. Temporal and B. Canaud, Eur. Phys. J. D 55, 139 (2009)

[12] J. Xiao and B. Lu, J. Opt. 29, 282 (1998)

[13] M. Temporal, B. Canaud, S. Laffite, B. Le Garrec and M. Murakami, Phys. Plasmas 17, 064504 (2010)

[14] M. Temporal, B. Canaud, and B. J. Le Garrec, Phys. Plasmas 17, 022701 (2010)

[15] B. J. Le Garrec, C. Hernandez-Gomez, T. Winstone, and J. Collier, J. Phys.: Conf. Ser. 244, 032020 (2010)

[16] M. Temporal, R. Ramis, B. Canaud, V. Brandon, S. Laffite, and B. J. Le Garrec, Plasma Phys. Control. Fusion 53, 124008 (2011)

[17] M. Temporal and B. Canaud, Eur. Phys. Journal D 65, 447 (2011)

[18] A. Schiavi, S. Atzeni, and A. Marocchino, EPL 94, 35002 (2011) 